\title{
Optogenetic Food Odor Avoidance Assay
}

Jay M. Patel ${ }^{1,2, \#, ~ J e s s i c a ~ S w a n s o n ~}{ }^{3, \#}$ and Benjamin R. Arenkiel ${ }^{1,2,3,4, *}$

${ }^{1}$ Department of Neuroscience, Baylor College of Medicine, Houston, USA; ${ }^{2}$ Medical Scientist Training Program, Baylor College of Medicine, Houston, USA; ${ }^{3}$ Department of Molecular and Human Genetics, Baylor College of Medicine, Houston, USA; ${ }^{4} \mathrm{Jan}$ and Dan Duncan Neurological Research Institute, Texas Children's Hospital, Houston, USA

*For correspondence: arenkiel@bcm.edu

\#Contributed equally to this work

[Abstract] Appetite is tightly linked to the sensory experience of feeding, including the smell, taste, and sight of food. Sensory perception can affect the palatability of food, modulating appetite beyond homeostatic requirements. Hypothalamic neurons that govern feeding are responsive to sensory cues associated with food, including food odors. However, the circuit mechanisms by which sensory information is processed and relayed to feeding nodes to affect feeding behavior is not well understood. Recent work has identified a population of excitatory basal forebrain neurons that modulate potent appetite suppression, as well as respond to food-associated and innately aversive odorants. To investigate this circuitry, we stereotaxically targeted virus expressing Cre-dependent channelrhodopsin to the basal forebrain and implanted fiber optic cannulas over the injection site. Mice were allowed to recover and underwent training to form a passive association of chow with a unique monomolecular odorant. After training, mice were fasted overnight, and were then presented with both the foodassociated odor as well as a similar, novel odor in zones of an arena with and without photostimulation. To evaluate whether stimulation of this circuitry influenced sensory modulation of feeding behavior, video recording and behavioral tracking analysis were used to compare time spent investigating either odor. Thus, this protocol provides a useful paradigm to assay the contribution of different circuits in appetitive and aversive behaviors.

Keywords: Mouse, Optogenetics, Olfactory, Feeding, Circuit, Neuroscience

[Background] Sensory cues can dramatically influence feeding behavior. Apart from taste, olfaction is a predominant sensory modality in feeding. The scent of food can override homeostatic mechanisms to influence or diminish the drive to eat ( $\mathrm{Li}$ and Liberles, 2015). Furthermore, olfaction has been shown to impact both food intake and metabolism (Riera et al., 2017). Interestingly, hypothalamic feedingassociated regions-such as the arcuate nucleus of the hypothalamus (ARC), lateral hypothalamus (LH), and paraventricular nucleus (PVN)-are modulated by olfactory perception of food (Betley et al., 2015; Chen et al., 2015; Jennings et al., 2015). Connected brain regions also integrate sensory information to regulate feeding. These regions include the anterior portion of the bed nucleus of the stria terminalis (aBNST), paraventricular thalamic nucleus (PVT), hindbrain (including the nucleus tractus solitarius), parabrachial nucleus, prefrontal cortex (PFC), amygdala, ventral tegmental area (VTA), and nucleus 
accumbens (NAc) (Waterson and Horvath, 2015; Rossi and Stuber, 2018). However, the circuit mechanisms by which olfactory information is relayed to the hypothalamus and other feeding-associated nodes are not well understood. To interrogate the circuitry relaying sensory cues to the hypothalamus, we must be able to manipulate circuit activity in a cell-type specific manner. Previous experiments have utilized a variety of methods to manipulate neurons to assay food intake such as toxin mediated ablation, and chemogenetics (Luquet et al., 2005; Herman et al., 2016; Stamatakis et al., 2016). Optogenetics allows for precise spatial and temporal control of neuronal activity when combined with conditional viral genetic approaches (Adamantidis et al., 2007; Jennings et al., 2013; Herman et al., 2016; Patel et al., 2019). Previous optogenetic assays have shown particular neural circuits are capable of modulating food intake and appetitive behaviors, however, they do not investigate the sensory component of feeding behaviors (Jennings et al., 2013; Herman et al., 2016).

To investigate how activation of basal forebrain neurons leads to hypophagia, we utilized optogenetics to activate the basal forebrain while recording how mice responded to food and food-associated odors (Patel et al., 2019). In this assay, optogenetic implanted mice were initially trained to form a passive association between a novel, neutral odorant and food. After training, animals were presented with the food-associated odor without photostimulation (stimulation control trial), the food-associated odor with photostimulation (experimental trial), or a completely novel odor with photostimulation (odor control trial). The odors were placed in three corners of a rectangular arena, leaving one corner empty. Additionally, mice were video-recorded, and these videos were analyzed post-hoc with video-tracking software to calculate the amount of time mice spent in each corner of the arena (Ben-Shaul, 2017). This allowed us to identify how a group of neurons mediated appetite suppression due to an aversion of food and foodassociated sensory cues (Patel et al., 2019).

\section{Materials and Reagents}

1. Fiber stripping tool (Thor Labs, catalog number: T10S13)

2. $200 \mu \mathrm{m}$ core multimode fiber, 0.22 NA (Thor labs, catalog number: FG200AEA)

3. $230 \mu \mathrm{m}$ bore size ceramic ferrules (Thor Labs, catalog number: CFLC230-10)

4. UV Light activated bonding tool with liquid plastic welder (Bondic, https://notaglue.com/)

5. Bare Fiber Gripper (Thor Labs, catalog number: BFG1)

6. Diamond knife (Fiber Instrument Sales, catalog number: F19773)

7. Instant readout digital caliper (Electron Microscopy Sciences, catalog number: 62065-40)

8. Polish disk (Thor Labs, catalog number: D50-FC)

9. $1.5 \mathrm{ml}$ tubes (Fisherbrand, catalog number: 05-408-129)

10. Cotton tipped applicators (Puritan, catalog number: $806-W C$ )

11. Polish paper (Diamond Lapping Sheet, $30 \mu \mathrm{m}-0.02 \mu \mathrm{m}$ ) (Thor Labs, catalog numbers: LF30D, LF6D, LF3D, LF1D, LFCF)

12. Rubber mat for polishing (Thor Labs, catalog number: NRS913A)

13. Stereotaxic Cannula Holder for $1.25 \mathrm{~mm}$ ferrules (Thor Labs, catalog number: $\mathrm{XCL}$ ) 
14. Surgical tools (dissection scissors, fine tweezers, hemostat, bulldog clamps)

15. 3.5" glass pipettes (Drummond, catalog number: 3000203G/X)

16. MicroFil 28 gauge/67 mm needle (World Precision Instruments, catalog number: MF28G67-5)

17. $1 \mathrm{ml}$ syringes (BD, catalog number: 309659$)$

18. 28,30 , and 18-gauge needles

19. Pre- and post- surgical heating pads (Sunbeam, 002011-905-622)

20. Alcohol/povidone-iodine swab ( $70 \%$ and $10 \%$ respectively)

21. Vacuum system for biological fluids

22. C\&B Metabond (Parkell, catalog number: 8750390)

23. Isofluorane (Henry Schein, catalog number: 029404)

24. Crosslinked flash acrylic (Yates and Bird 44115 \& 44119)

25. $70 \%$ ethanol and/or disinfectant solution

26. Ophthalmic ointment (Celluvisc)

27. AAV (serotype 2/9), tittered to $1 \times 10^{12}$ infectious particles $/ \mu \mathrm{l}$
a. For experimental optogenetic animals: pAAV-DIO-hChR2(H134R)-EYFP-WPRE-PA (or similar)
b. For control optogenetic animals: pAAV-Ef1a-DIO-GFP (or similar)

28. Meloxicam ( $5 \mathrm{mg} / \mathrm{ml}$, diluted to working concentration of $0.5 \mathrm{mg} / \mathrm{ml}$; dose required is $5 \mathrm{mg} / \mathrm{kg}$. Henry Schein Inc., catalog number: 049755)

29. Sterile saline (Farris laboratories, catalog number: 00409-4888-20)

30. Mineral oil (Alfa Aesar, catalog number: 31911)

31. Odorant pairs for trained and novel odorant: $\mathrm{R}-(+)$-Limonene (Sigma-Aldrich, catalog number: 183164) and S-(-)-Limonene (Sigma-Aldrich, catalog number: 218367), or Methyl acetate (Sigma-Aldrich, catalog number: W267600) and Ethyl acetate (Sigma-Aldrich, catalog number: 34858)

\section{Equipment}

1. Leica Angle Two Computer-Assisted stereotaxic system

2. Nanoject II Auto-Nanoliter Injector (Drummond Scientific, catalog number: 3-000-204)

3. Zeiss Stemi 2000 Stereoscope

4. Tool Sterilizer (Germinator500; Braintree Scientific, Inc., catalog number: GER 5287-120V)

5. Vaporizer for isoflurane administration (Vetequip, catalog number: 911103)

6. Glass pipette puller (Sutter Instruments, model: P-30)

7. LD fiber light source (Doric, catalog number: LDFLS_405/100)

8. FC-to-FC cables (Doric, catalog number: MFP-200/220/900-0.22_1.25_FC_FC)

9. Rotary joint (Doric, catalog number: FRJ_FC-FC)

10. FC-to-ferrule patch cables (Doric, catalog number: BFP(2)_200/220/900-0.22_1.25_FCM-2xFC)

11. Optical power meter (Thorlabs, catalog number: PM20A) 
12. Behavior arena (18 Qt Clear Square Polycarbonate Food Storage Container, WebstaurantStore, catalog number: $176 S Q R C L 18)$

13. Behavior tracking video camera (Doric, catalog number: BTC_GigE_CO)

14. Computer (Dell or similar)

15. Handheld drill (Vector Mega Torque with $0.5 \mathrm{~mm}$ dental drill bit, model: EL-S)

\section{Software}

1. Doric Neuroscience Studio (Doric Lenses, http://doriclenses.com/life-sciences/software/955doric-neuroscience-studio.html)

2. MATLAB (Mathworks, www.mathworks.com)

3. Optimouse (Ben-Shaul, 2017, https://github.com/yorambenshaul/optimouse)

\section{Procedure}

A. Fiber optic creation (Figures $1 \mathrm{~A}$ and $1 \mathrm{~B}$ )

1. Cut $\sim 4-5$ inches of the optic fiber, providing you with ample material to work with for your implant.

2. Strip 2" of optic fiber with T10S13 micro-strip stripping tool. The exact length doesn't matter, as long as it's longer than the length of the ceramic ferrule, which is $6.4 \mathrm{~mm}$.

3. Line the stripped part of fiber with the Bondic liquid plastic welder, and insert the optic fiber into the large bore side of the ceramic ferrule. Slide the stripped fiber through the ferrule until it naturally comes to a stop at the non-stripped portion of the fiber.

4. Secure the optic fiber in the ceramic ferrule by curing the liquid plastic with UV light. This takes about 2 min.

5. Cleave off the excess stripped fiber from the small-bore end of the implant using a diamond knife. To cleave, gently score the fiber perpendicular to the implant, and then pull off excess fiber using the bare fiber gripper.

6. Cleave the optic fiber at the large bore end, which is the portion of the fiber optic that is inserted into the brain, to the correct length for your implant site using a diamond knife. Ensure the length is approximately $300 \mu \mathrm{m}$ above the target brain region. In other words, the total length of the non-stripped fiber $=\mathrm{x} \mu \mathrm{m}-300 \mu \mathrm{m}$ (where $\mathrm{x}$ is the appropriate target depth). For example, to target a region $3 \mathrm{~mm}$ ventral from the skull, the fiber optic should measure $2.7 \mathrm{~mm}$. Measure the length accurately using a caliper. Best cleavage is obtained by gently scoring the fiber perpendicularly, and then pulling straight away from the cleavage site, causing the excess fiber to "pop" off. After cleavage, this end of the fiber should be a smooth, flat circle without shards or cracks. Poor cleavage will result in poor light output, as this end of the implant cannot be polished.

7. Using a polishing disk and a hemostat to hold the implant in place, polish the ferrule tip (smallbore end) on polishing paper (placed on top of the rubber polishing mat) proceeding from high 
to low grit ( $30 \mu \mathrm{m}$ down to $0.02 \mu \mathrm{m})$ in a Figure 8 motion. Spend about 1-2 min on each grit. The higher grit polish paper is used to fix and chips or shards in the fiber, creating a flat surface. Once the surface of the fiber is smooth and level, progress in descending grit order to continue polishing the fiber, ensuring maximum light output by going from a rough surface to a smooth surface. The small-bore end of the fiber should be very shiny and clear once it is properly polished.

8. Confirm transmittance of the optic fiber. Check the shape of the laser beam and power output. The shape of the laser beam should be a circle, and if it is polished nicely you will even see concentric circles (Figure 1B). Depending on the light source/power used, the minimum light output should be $1 \mathrm{~mW}$. You can check light output using an optical power meter.

A

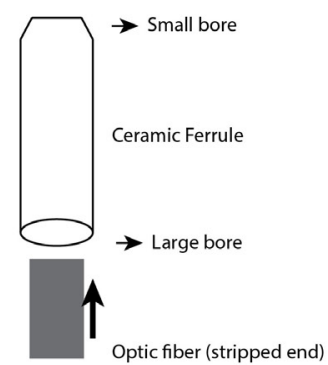

B

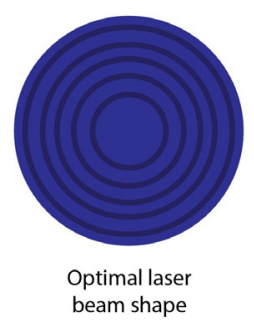

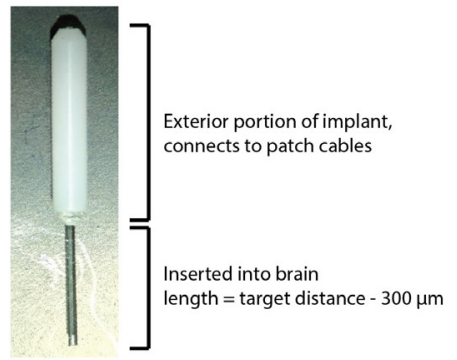

length $=$ target distance $-300 \mu \mathrm{m}$
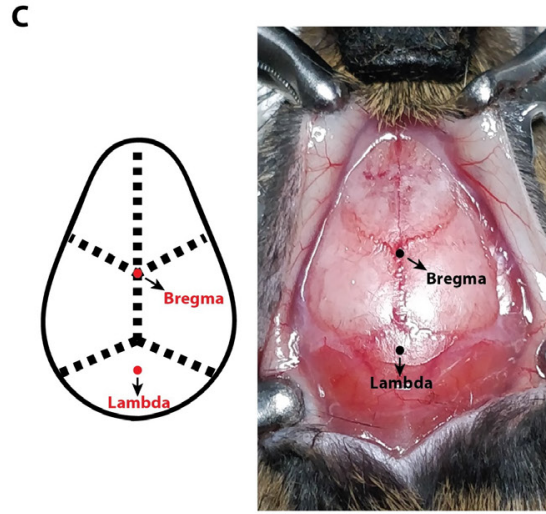

D

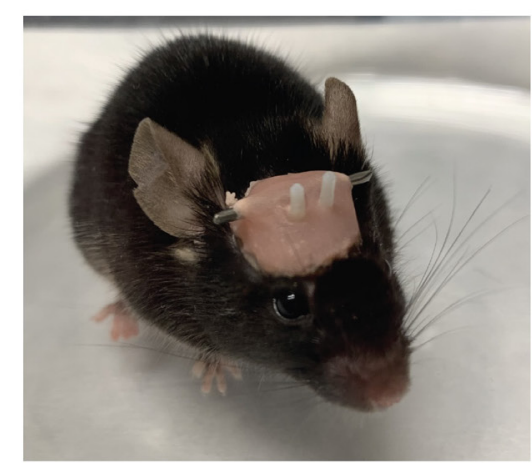

Figure 1. Fiber Optic implantation in Mouse. A. Ceramic ferrule orientation for optic fiber and example final optic fiber implant. B. Optimal laser beam shape after polishing is a clean circle with high light output, and if polished and cleaved well, contains concentric circles. In comparison, poor laser beam shapes are fuzzy or misshaped circles with lower light output. C. Mouse skull exposed with diagram indicating bregma and lambda for stereotaxic injection. D. Mouse with bilateral fiber optic implants, pink skull cap (acrylic), and metal holding bar (18G needle). 


\section{B. Surgery preparation}

1. Sterilize surgery area using $70 \%$ ethanol, and sterilize surgical instruments using disinfectant (Germinator 500 ) followed by $70 \%$ ethanol.

2. Treat mouse with $5 \mathrm{mg} / \mathrm{kg}$ meloxicam and $10 \mathrm{mg} / \mathrm{kg}$ sterile saline subcutaneously $30 \mathrm{~min}$ prior to surgery. Repeat meloxicam injection every $24 \mathrm{~h}$ for a minimum of $72 \mathrm{~h}$ while monitoring animal for signs of pain or distress.

3. Prepare your micropipette for injection. Pull a 3.5" glass pipette with a glass pipette puller, with the tip being about $1 \mathrm{~cm}$ long. Once pulled, snip off about $1 \mathrm{~mm}$ of the glass tip with surgical scissors to ensure the end of the tip is open. Back-fill the glass pipette using a MicroFil 28 gauge/67 mm needle and $1 \mathrm{ml}$ syringe filled with sterile mineral oil to prevent contamination, and load the pipette onto the Nanoject II.

4. Empty the pipette of mineral oil and then re-fill with the desired volume of virus, depending on your region of interest. Small regions may require as little as $20 \mathrm{nl}$, while larger regions may require $500 \mathrm{nl}$ or more.

5. Weigh the mouse and sedate with sufficient isoflurane ( $1-4 \%$ isoflurane diluted in $100 \%$ oxygen), monitoring vitals of the animal.

6. Shave the mouse's scalp and clean with alcohol/povidone-iodine swab ( $70 \%$ and $10 \%$ respectively).

7. Place the animal on the stereotaxic rig by hooking their front incisor teeth into the mouth bar. Straighten the animal, and then fix the ear bars into the ear canals. Once secure, tighten the nose bar firmly over the nose, without blocking airflow.

8. Maintain the animal under anesthesia using $1-3 \%$ isoflurane diluted with $100 \%$ oxygen using a Vetequip vaporizer. The dosage of isoflurane depends on the physiological state of the animal as monitored by response to tail pinch.

9. Maintain proper thermoregulation during surgery by using a heat pad set to $37^{\circ} \mathrm{C}$. Apply ophthalmic ointment to mouse's eyes to prevent dryness and discomfort during surgery.

C. Viral vector injection (Figure 1C)

1. Make an incision in the scalp with surgical scissors at the posterior end of the brain, and cut anteriorly towards the olfactory bulb in a straight line.

2. Use bulldog clamps to pull the skin away from the skull, exposing the skull. Keep the skull moist during surgery with sterile saline.

3. To calibrate the Leica Angle Two Stereotax system, locate Bregma and then lower the tip of the micropipette, mounted onto the Nanoject II on the stereotaxic holder, to rest lightly on it. Then click "at Bregma" in the software. After identifying Bregma, raise the needle, and check the $z$ coordinates by moving $1.5 \mathrm{~mm}$ to the left and right of Bregma, lowering the micropipette to touch the skull carefully, and take note of the $z$ coordinate. The skull will need to be adjusted until the tilt is level, with a margin of error of $\pm 0.03 \mathrm{~mm}$. 
4. After establishing the medial-lateral axis using Bregma, Lambda is used to establish the anterior-posterior axis. Once again, lower the micropipette to lambda and take note of the $z$ coordinate. Adjust the skull until the dorsal/ventral coordinates of Lambda and Bregma are within $\pm 0.03 \mathrm{~mm}$ of each other, selecting "At Lambda" once level.

5. Identify your injection site using coordinates from the Allen Brain Atlas (installed in the Leica Angle Two software). It is best to validate coordinates empirically before beginning an experiment.

6. Make a small burr hole craniotomy $(<1 \mathrm{~mm})$ over the injection site using a hand-held dental drill with a $0.5 \mathrm{~mm}$ drill bit. Clear bits of skull away from the center of the craniotomy.

7. Lower the micropipette slowly to the injection site $(\sim 3 \mathrm{~mm} / \mathrm{min})$ and upon arrival at the target site, wait 5 min before injecting virus to allow the brain to settle. Inject discrete volumes of virus every $30 \mathrm{~s}$ (100 $\mathrm{nl}$ or less) using the Nanoject II. Once the total volume of virus has been injected, wait another $5 \mathrm{~min}$ before slowly raising the needle out of the brain.

Note: Ensure that the micropipette does not become clogged or break during the surgery. To check for a clogged micropipette, eject a small volume of virus before lowering the micropipette into the brain, and ensure that virus flows out of the micropipette. If no virus appears, snip the end of the micropipette and re-calibrate Bregma.

8. If you are doing bilateral injections, repeat Procedure $C$ for the opposite side. Keep skull moist during injections using sterile saline.

D. Fiber implantation

1. Remove the Nanoject II from the stereotaxic frame and attach a stereotaxic cannula holder onto frame.

2. Etch a checkered pattern on skull surface with a 28 gauge needle. This "scoring" of the skull increases surface area for cement to bind, increasing durability of the implant. Wash any debris away with sterile saline and dry thoroughly. The skull must be dry for cement to bind.

3. Insert the fiber optic cannula into the cannula holder.

4. Locate Bregma with the fiber optic implant and select "At Bregma" once more. Then go to the coordinates of your injection site. If the fiber optic implant site is different from the injection site, drill holes over the target stimulation site(s).

5. Lower the fiber optic implant to the target stimulation site. Lower the implant at a slow rate of $1-2 \mathrm{~mm} / \mathrm{min}$.

6. In one well of a cold ceramic plate, place 4 drops of Metabond solvent and 1 drop of Metabond catalyst and mix well.

7. Apply the liquid solution from well \#1 to the base of the ceramic ferrule and the skull surface using a sterile applicator.

8. In another well (\#2), place 4 drops of Metabond solvent, 2 scoops of Metabond cement powder, and 1 drop of Metabond catalyst. Mix well. 
9. Apply a thin, even layer of dental cement (well \#2) across the skull and lower portion of the implant, creating support around the base of the implant. Continue applying even layers until the cement in well \#2 is used up.

10. Allow dental cement to dry completely.

11. Repeat Steps D3-D10 for the second implant if doing bilateral implants.

Note: You will not be able to re-establish Bregma; thus, guide the implant through the second craniotomy and calculate the required distance in the $z$ direction to reach the stimulation target using the Angle 2 software.

12. Cut an 18 gauge needle to $1.5 \mathrm{~cm}$ in length using wire cutters at each end. Squeeze ends with a plier to dull them.

13. Combine liquid and powder components of cross-linked flash acrylic to create a liquid jelly.

14. Apply a final, even acrylic layer over the dental cement, ensuring the implants are well-secured. Build a mound of acrylic at the posterior end of the skull, placing the clipped 18 gauge needle at this position and securing it in place with further layers of acrylic. This will allow you to use ring-end forceps to grasp the mouse without having to scruff the animal (Figure 1D).

15. Allow the mouse to recover in a clean cage on a heating pad. Monitor the animal every $15 \mathrm{~min}$ for $4 \mathrm{~h}$, looking for signs of pain or distress. If they are not recovering as expected, euthanize humanely.

\section{E. Passive odor association}

1. After one day of recovery, begin passive odor association, which is where mice learn to associate food with a specific odorant. Choose two odors to be used in the experiment: one for association with food, and the other one as a novel odorant that will serve as a control during testing. If possible, you may want to choose an odor that has an enantiomer and is neither appetitive, nor aversive, or a "neutral" odorant (Devore et al., 2013). One good odor pair is R and S-Limonene, which are enantiomers. Methyl and ethyl acetate may also work well, as they have similar structures and are relatively neutral.

2. Dissolve odorant to be associated with food in $5 \mathrm{ml}$ of mineral oil to create a $2 \%$ by volume odor mixture. Mineral oil is odorless, so it is only used to dilute the concentrated odorant.

3. Using a dropper, place $2 \mathrm{ml}$ of odor mixture onto roughly $15 \mathrm{~g}$ of mouse chow pellets. Add odorspiked chow to feeding hatch, or wherever the mouse normally receives food.

4. Change out food with fresh odorant every 2 days, repeating for a total of 2 weeks. Use the same odorant throughout the training process. House mice in their normal home cage during this association process.

F. Behavior assay habituation-6 days

Note: This can be completed during passive food odor association.

1. Create a behavior box ( $11 \times 11 \times 12$ inches) with 4 corners containing a $1.5 \mathrm{ml}$ tube secured to the wall with tape (Figure 2B). 
2. Place a cotton swab in each $1.5 \mathrm{ml}$ tube (you may need to alter the length to fit in the $1.5 \mathrm{ml}$ tube). (Figure 2A)

3. Place animals in the behavior box arena for 30 min for 3 days.

4. Place animals in the behavior box arena for $30 \mathrm{~min}$ for 3 days while being tethered to fiber optic patch cords.
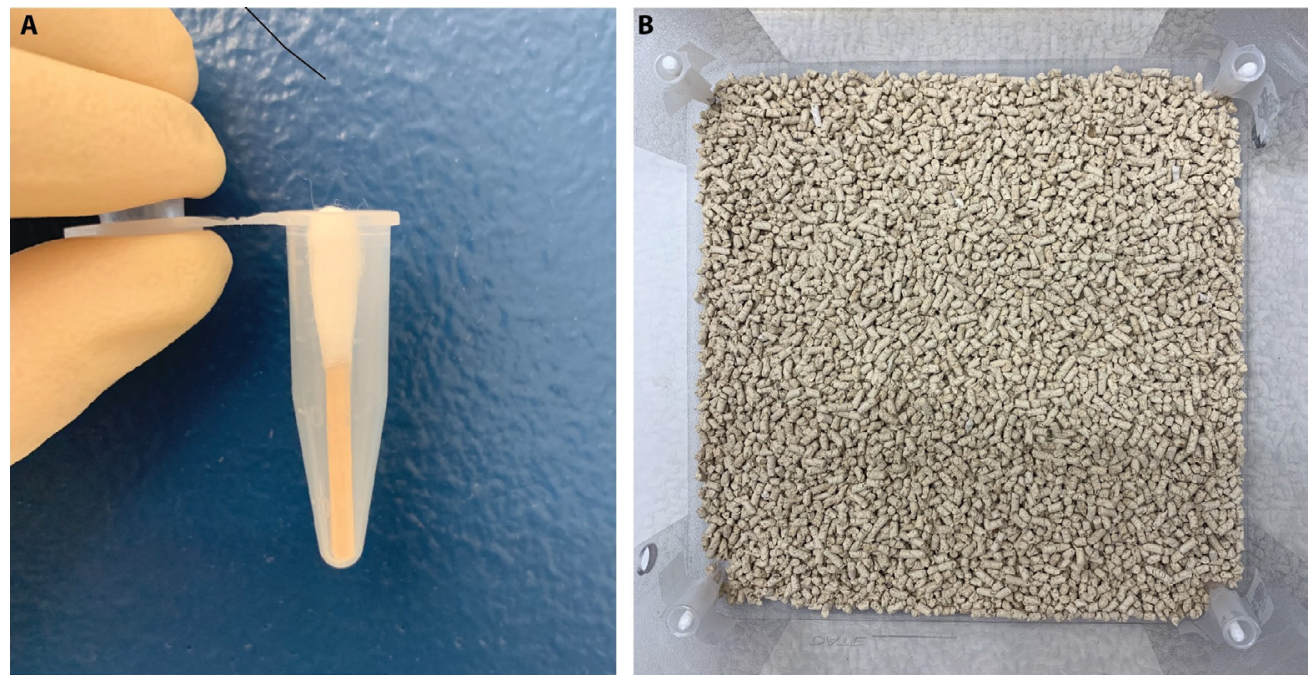

Figure 2. Behavior box construction. A. Cotton swab trimmed to the appropriate length and placed in a $1.5 \mathrm{ml}$ tube for future odor presentation. B. Four odor presentation tubes taped to the 4 corners of a square behavior box.

G. Behavior assay testing (Figure 3)

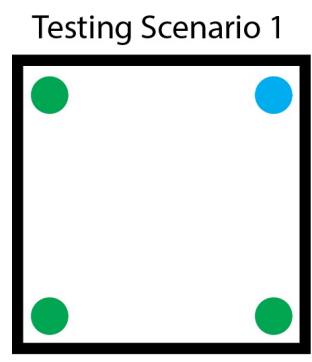

No Photostimulation

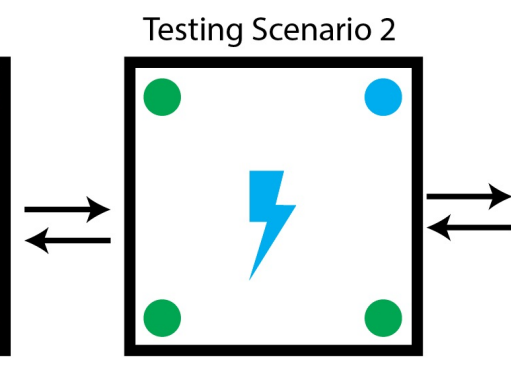

Photostimulation
Testing Scenario 3

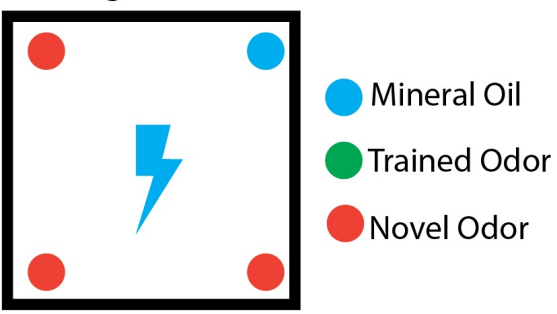

Photostimulation

Figure 3. Behavior testing paradigm. Testing paradigms indicating odor placement and photostimulation for each scenario.

1. For the testing phase, you will compare the time optogenetically-implanted mice spend investigating the food-associated odor with and without photostimulation. This is to observe the effect of activating a neuronal population of interest on food odor processing, as food odors are normally appetitive. As a control, animals are also presented a novel, non-trained odor during photostimulation, to ensure that the difference in investigation time during photostimulation is specific to food odors alone. For testing, divide mice into 2 groups: Group A and Group B. Group 
A animals will go through testing in order 1, 2, 3, while group B will go in reverse order 3, 2, 1. This is to prevent habituation to odors. Randomize mice to these two groups by numbering mice and using a randomization generator (available in Graphpad). Have a labmate number each mouse so that the experimenter is blind during the experiment and analysis. Mice are fasted overnight prior to testing and are allowed to rest for $5 \mathrm{~min}$ in the behavior arena between testing scenarios.

\section{Test Scenario \#1}

Cotton Swabs are dipped in mixture of mineral oil + food associated odor ( $2 \%$ by volume) and placed in 3 of the 4 tubes. One tube has a cotton swab dipped in mineral oil only, which is the odorless control. Mice are attached to patch cords and placed in the behavior arena for $10 \mathrm{~min}$ while being video recorded.

3. Test Scenario \#2

Same as \#1 however mice are photostimulated at a frequency ideal for activation of the neuronal population of interest. Mice are video recorded for 10 min during this photostimulation.

\section{Test Scenario \#3}

Same as scenario \#2, however three cotton swabs are dipped in $2 \%$ mixture by volume of a novel, non-conditioned odorant. It may be helpful to choose an odorant that is similar to the food-associated odor. Mice are video recorded for $10 \mathrm{~min}$ during this photostimulation.

5. During all scenarios, video record mice for post-hoc tracking analysis. Utilize a minimum video recording rate of $4 \mathrm{~Hz}$. Higher rates can be used for finer detection, but will require greater computer memory and processing power. Ensure cages are evenly lit and videos have enough contrast that mice can easily be seen and that there are no large shadows in the cages.

\section{Data analysis}

Data analysis is completed using the free, open source plug-in software "Optimouse" run in MATLAB. Briefly, videos are opened in the Optimouse plug-in.

1. The first step of the program involves preparation by drawing arenas around behavior cages to isolate individual cages, allowing for multiple cages to be video recorded at once.

2. After arenas are prepared, utilize the software to detect mice. Different parameters can be changed to ensure proper and accurate mouse detection. For example, altering the threshold (increasing it for increased stringency and decreasing it for decreased stringency) and peeling steps can improve the detection parameters.

Note: If the video has uneven lighting or other issues preventing proper detection, different detection parameters can be used in different frames to achieve optimal detection. This is done in the next step.

3. After detection, the mouse position can be reviewed to ensure proper identification. Ensure proper mouse identification at a rate of at least $95 \%$ (i.e., mouse was correctly identified in $95 \%$ of frames and missed in only up to $5 \%$ of frames). Alter mouse detection algorithms to achieve 
this rate.

4. After arena preparation and mouse detection, videos can be analyzed. Regions of interest can be drawn in corners with odors, and the amount of time spent in the regions can be extracted. We calculated the percentage of time spent in the non-odor corner across each of the three test scenarios and performed a repeated measures one-way ANOVA, post-hoc Tukey to compare test scenario 2 to control test scenarios 1 and 3 . This allowed each mouse to be its own control (i.e., by comparing odor investigation time while being stimulated to odor investigation time without stimulation). More information about Optimouse can be found in Ben-Shaul, 2017. Additional information on data analysis and data presentation can be found in Figure 10 and Materials and methods in Patel, et al. (2019).

\section{$\underline{\text { Notes }}$}

Please refer to the original publication for more applications of this protocol. https://elifesciences.org/articles/44548.

\section{Acknowledgments}

The project described was supported through the multi-PI award R01DK109934 to BRA and QT, and the Neuroconnectivity Core at Baylor College of Medicine, which is supported by IDDRC Grant Number 1 U54 HD083092 from the Eunice Kennedy Shriver National Institute of Child Health and Human Development. The content is solely the responsibility of the authors and does not necessarily represent the official views of the Eunice Kennedy Shriver National Institute of Child Health and Human Development or the National Institutes of Health. The project was supported by F30DK112571 to JMP. We would also like to thank the McNair Medical Institute and Charif Souki for their ongoing and generous support. We would like to acknowledge that parts of this technique were originally described by Sparta et al., 2012, Ung et al., 2012, and Patel et al., 2019.

\section{Competing interests}

The authors declare that no competing interests exist.

\section{Ethics}

Animal experimentation: All mice in this study were treated in compliance with US Department of Health and Human Services and Baylor College of Medicine and in strict accordance with the recommendations in the Guide for the Care and Use of Laboratory Animals of the National Institutes of Health. All of the animals were handled according to approved institutional animal care and use committee (IACUC) protocol (AN5966) at Baylor College of Medicine. All surgery was performed in 
accordance with the approved protocol, and every effort was made to minimize suffering.

\section{References}

1. Adamantidis, A. R., Zhang, F., Aravanis, A. M., Deisseroth, K. and de Lecea, L. (2007). Neural substrates of awakening probed with optogenetic control of hypocretin neurons. Nature 450(7168): 420-424.

2. Ben-Shaul, Y. (2017). OptiMouse: a comprehensive open source program for reliable detection and analysis of mouse body and nose positions. BMC Biol 15(1): 41.

3. Betley, J. N., Xu, S., Cao, Z. F. H., Gong, R., Magnus, C. J., Yu, Y. and Sternson, S. M. (2015). Neurons for hunger and thirst transmit a negative-valence teaching signal. Nature 521(7551): 180-185.

4. Chen, Y., Lin, Y. C., Kuo, T. W. and Knight, Z. A. (2015). Sensory detection of food rapidly modulates arcuate feeding circuits. Cell 160(5): 829-841.

5. Devore, S., Lee, J. and Linster, C. (2013). Odor preferences shape discrimination learning in rats. Behav Neurosci 127(4): 498-504.

6. Herman, A. M., Ortiz-Guzman, J., Kochukov, M., Herman, I., Quast, K. B., Patel, J. M., Tepe, B., Carlson, J. C., Ung, K., Selever, J., Tong, Q. and Arenkiel, B. R. (2016). A cholinergic basal forebrain feeding circuit modulates appetite suppression. Nature 538(7624): 253-256.

7. Jennings, J. H., Rizzi, G., Stamatakis, A. M., Ung, R. L. and Stuber, G. D. (2013). The inhibitory circuit architecture of the lateral hypothalamus orchestrates feeding. Science 341(6153): 15171521.

8. Jennings, J. H., Ung, R. L., Resendez, S. L., Stamatakis, A. M., Taylor, J. G., Huang, J., Veleta, K., Kantak, P. A., Aita, M., Shilling-Scrivo, K., Ramakrishnan, C., Deisseroth, K., Otte, S. and Stuber, G. D. (2015). Visualizing hypothalamic network dynamics for appetitive and consummatory behaviors. Cell 160(3): 516-527.

9. Li, Q. and Liberles, S. D. (2015). Aversion and attraction through olfaction. Curr Biol 25(3): R120-R129.

10. Luquet, S., Perez, F. A., Hnasko, T. S. and Palmiter, R. D. (2005). NPY/AgRP neurons are essential for feeding in adult mice but can be ablated in neonates. Science 310: 683-685.

11. Patel, J. M., Swanson, J., Ung, K., Herman, A., Hanson, E., Ortiz-Guzman, J., Selever, J., Tong, Q. and Arenkiel, B., R. (2019). Sensory perception drives food avoidance through excitatory basal forebrain circuits. Elife 8: e44548.

12. Riera, C. E., Tsaousidou, E., Halloran, J., Follett, P., Hahn, O., Pereira, M. M. A., Ruud, L. E., Alber, J., Tharp, K., Anderson, C. M., Bronneke, H., Hampel, B., Filho, C. D. M., Stahl, A., Bruning, J. C. and Dillin, A. (2017). The sense of smell impacts metabolic health and obesity. Cell Metab 26(1): 198-211 e195.

13. Rossi, M. A. and Stuber, G. D. (2018). Overlapping brain circuits for homeostatic and hedonic feeding.Cell Metab 27(1): 42-56. 
Please cite this article as: Patel et. al., (2019). Optogenetic Food Odor Avoidance Assay,Bio-protocol 9 (20): e3406. DOI: 10.21769/BioProtoc.3406.

14. Sparta, D. R., Stamatakis, A. M., Phillips, J. L., Hovelso, N., van Zessen, R. and Stuber, G. D. (2011). Construction of implantable optical fibers for long-term optogenetic manipulation of neural circuits. Nat Protoc 7(1): 12-23.

15. Stamatakis, A.M., Van Swieten, M., Basiri, M.L., Blair, G.A., Kantak, P., and Stuber, G.D. (2016). Lateral hypothalamic area glutamatergic neurons and their projections to the lateral habenula regulate feeding and reward. J Neurosci Off J Soc Neurosci 36:302-311.

16. Ung, K. and Arenkiel, B.R. (2012). Fiber-optic implantation for chronic optogenetic stimulation of brain tissue. $J$ Vis Exp JoVE e50004.

17. Waterson, M. J. and Horvath, T. L. (2015). Neuronal regulation of energy homeostasis: beyond the hypothalamus and feeding. Cell Metab 22(6):962-970. 